\title{
Fuzzy Implication and Functional Dependency on Formal Context
}

\author{
Mohammad Deni Akbar ${ }^{1}$, Yoshiniro Mizoguchi ${ }^{2}$ \\ ${ }^{1}$ School of Industrial Engineering, Telkom University \\ ${ }^{2}$ Institute of Mathematics for Industry, Kyushu University
}

\begin{abstract}
Fuzzy formal concept analysis(FFCA) is a development of formal concept analysis(FCA) with the degree of relation between objects and attributes. Using FCA approach, we will investigate the condition logical implication for fuzzy functional dependency. We also use Armstrong's rule to define soundness and completeness of our implication and fuzzy functional dependency model. We show difference and equivalence condition between fuzzy implication and fuzzy functional dependency. This condition can be used to develop the algorithm for finding attribute dependency.

Keywords:Formal Concept Analysis, Fuzzy Implication, Fuzzy Functional Dependency.
\end{abstract}

\section{INTRODUCTION}

Dependency theory is an essential theory in data analysis. A notion of functional dependency, which is a constraint between two sets of attributes has been introduced by Codd [10]. Model of attribute dependency has been developed by Belohlvek et al. $[7,8,6]$.

In the real condition, it is difficult to give "certain" value for representing the condition. We have to deal "uncertain" condition with the "degree". For example, we give value from 0 to 1 to define the condition "Healthy Food", "Regularly Exercise", "Cholesterol"

\begin{tabular}{|c|c|c|c|}
\hline Name & Healthy Food & Regularly Exercise & Cholesterol \\
\hline Asep & 0.8 & 0.7 & 0.2 \\
Ujang & 0.3 & 0.7 & 0.5 \\
Paijo & 0.1 & 0.3 & 0.87 \\
\hline
\end{tabular}

TABLE 1. List of Patients

From table 1, we use "degree" value to define high possibility of the condition. We would like to find the correlations between attributes. We have to use fuzzy system to define the correlation. From table 1, we can suspect that "Healthy Food" and "Regularly Exercise" have impact to the "Cholesterol ((Healthy Food, Regularly Exercise) $\rightarrow$ Cholesterol). In this research, we would like to investigate the dependency and implication in fuzzy case.

In the first section, we formalize fuzzy relation from fuzzy theory that has been introduced by Zadeh[19]. The Fuzzy relational database is a development model of the relational database to process imprecise data. Fuzzy relational database and its operation have been developed by Nakata et al.[14], Raju[16], and Umano et al.[17]. Formal operation of the fuzzy relational

2000 Mathematics Subject Classification: 03B52

Received: 2019-09-26, Revised: accepted: 2020-01-21; 2020-02-01. 
database using relational calculus has been developed by Okuma et al.[15], Akbar et al.[1, 2]. After defining model of the fuzzy relational database, fuzzy functional dependency and lossless decomposition have been defined by Raju[16], and Bhuniya[9]. Class dependency is a new dependency using fuzzy partition and conditional probability. Fuzzy class dependency has been developed by Akbar et al. [3].

Ganter and Wille defined logical implication for a formal concept analysis[12]. Formal concept analysis is a method in data mining using lattice theory proposed by Wille[18]. Ishida et al. have been developed a formalization of completeness and soundness for functional dependencies based on [5] using relational calculus[13]. They showed a relation-algebraic proof of the completeness theorem for Armstrong's inference rules in a Schröder category. Comparing formalization to a functional dependency and a logical implication of FCA was also investigated. We extend its formalization using a fuzzy relational concept. A notion of fuzzy was initially proposed by Zadeh[19]. In section 5, We define fuzzy equivalence relation using an indiscernibility relation by Düntsch and Günther[11] to construct a fuzzy functional dependency in section 7. In section 6 , we formalized a fuzzy implication. Then in section 8 , we give the Completeness theorem for fuzzy implication and fuzzy functional dependency. In section 9, The main purpose of our formalization is investigating correlations between a functional dependency and a logical implication of FCA. We follow the Ishida's approach to defining Armstrong's inference rules that explain in section 4, and show the soundness and completeness in our formalization using a fuzzy relation.

We introduce the formalization of the fuzzy equivalence relation, fuzzy implication dependency, and functional dependency. The formalization can be used to analyze the equivalent condition between functional and implication. We prove theorems in our formalization of fuzzy concepts using relational calculus. Since our proof is using relational calculus, it is simple and its correctness can be easily verified. We also show the logical comparison between fuzzy implication dependency and functional dependency. Further, the comparison is important aspects for making the algorithm of fuzzy functional and implication dependency. We show some examples of the application of data analysis. Since formal concept analysis is essential theory in data analysis, we believe our formalization useful for data analysis. Future work includes to constructing a theory of fuzzy relational database theory with computer verified formal proofs using relational calculus.

\section{Fuzzy Relations}

In this section, we summarize basic notations for fuzzy relations. We denote the set $\{\mathbf{x} \in \mathbb{R} \mid 0 \leq \mathbf{x} \leq 1\}$ as $[0,1]$. The supremum and infimum of a family $\left\{x_{\lambda}\right\}_{\lambda \in \Lambda}$ of elements $x_{\lambda} \in[0,1]$ is denoted by $\bigvee_{\lambda \in \Lambda} x_{\lambda}$ and $\bigwedge_{\lambda \in \Lambda} x_{\lambda}$, respectively. In particular, $x \vee x^{\prime}=\max \left\{x, x^{\prime}\right\}$, $x \wedge x^{\prime}=\min \left\{x, x^{\prime}\right\}$ for $x, x^{\prime} \in[0,1]$. For two elements $x, x^{\prime} \in[0,1]$, the relative pseudocomplement $\Rightarrow$ of $x$ relative to $x^{\prime}$ defined by $x \Rightarrow x^{\prime}:=\left[x \leq x^{\prime}\right] \vee x^{\prime}$, where

$$
\left[x \leq x^{\prime}\right]:= \begin{cases}1 & \text { if } x \leq x^{\prime} \\ 0 & \text { otherwise }\end{cases}
$$

Lemma 2.1. (a) Let $x, y, z \in[0,1]$ then $x \leq(y \Rightarrow z)$ if and only if $x \wedge y \leq z$.

(b) Let $\alpha, \beta, \gamma$ be fuzzy relations, $\alpha, \beta, \gamma: X \rightarrow Y$ then $\alpha \sqsubseteq(\beta \Rightarrow \gamma)$ if and only if $\alpha \sqcap \beta \sqsubseteq \gamma$.

We extend to define fuzzy operations $\sqcup$ (union), $\sqcap$ (intersection), $\sqsubseteq$ (subset), $\Rightarrow$ (the relative pseudo-complement), and constants $\mathbf{0}_{A B}$ (least), $\nabla_{A B}$ (greatest) in $F R e l$, as follows:

(1) $(\alpha \sqcup \beta)(a, b):=\alpha(a, b) \vee \beta(a, b)$,

(2) $(\alpha \sqcap \beta)(a, b):=\alpha(a, b) \wedge \beta(a, b)$,

(3) $\alpha(a, b) \sqsubseteq \beta(a, b)$ iff $\alpha(a, b) \leq \beta(a, b)$,

(4) $\mathbf{0}_{A B}(a, b):=0$, and

(5) $\nabla_{A B}(a, b):=1$. 
The fuzzy power set $\wp_{f}(Y)$ of a set $Y$ is the set of all fuzzy relations $\rho: I \rightarrow Y$, where $I$ denotes a singleton set $\{*\}$. A fuzzy relation $\rho$ in $\wp_{f}(Y)$ is called a fuzzy relation into $Y$. We will identify a point $y$ of $Y$ with a (crisp) fuzzy relation $\hat{y}: I \rightarrow Y$ such that $\hat{y}\left(*, y^{\prime}\right)=1$ if $y^{\prime}=y$ and $\hat{y}\left(*, y^{\prime}\right)=0$ otherwise.

Let $B: I \rightarrow Y$ be a fuzzy relation and $y \in Y$. The restriction $B_{y}$ of $B$ on $y$ is a fuzzy relation into $Y$ such that

$$
B_{y}\left(*, y^{\prime}\right)= \begin{cases}B(*, y) & y^{\prime}=y, \\ 0 & \text { Otherwise }\end{cases}
$$

It is trivial that $B=\sqcup_{y \in Y} B_{y}$.

A fuzzy relation $B: I \rightarrow Y$ is finite if $B_{y}=0_{I Y}$ except for a finite number of $y \in Y$, or equivalently if there is a finite subset $J \subseteq Y$ such that $B=\sqcup_{y \in J} B_{y}$.

\section{Fuzzy Context}

Attribute values in ordinary databases may allow to be characters or strings as well as numbers. A context is a special database whose all attribute values are the truth values 0 and 1. A fuzzy context is a fuzzy relation $\alpha: X \rightarrow Y$, which is equivalent to its intent function $\alpha^{@}: X \rightarrow \wp(Y)$ defined as $\alpha^{@}(x)=x \alpha$ for all $x \in X$. Thus the fuzzy context $\alpha: X \rightarrow Y$ is equivalent to an $\mathrm{X}$-indexed set $\tau=\{x \alpha \mid x \in X\}$ of fuzzy relations into $Y$, where $x \alpha$ denotes the composite of fuzzy relations $x: I \rightarrow X$ and $\alpha: X \rightarrow Y$.

\begin{tabular}{|c|c|c|c|l|c|}
\hline & $y_{1}$ & $y_{2}$ & $y_{3}$ & $\cdots$ & \\
\hline$x_{0}$ & 0.1 & 0.5 & 0 & $\cdots$ & $x_{0} \alpha$ \\
$x_{1}$ & 0.9 & 1 & 0.3 & $\cdots$ & $x_{1} \alpha$ \\
$x_{2}$ & 0 & 0.7 & 0.2 & $\cdots$ & $x_{2} \alpha$ \\
$\vdots$ & $\vdots$ & $\vdots$ & $\vdots$ & $\vdots$ & $\vdots$ \\
$x_{n}$ & 0.4 & 1 & 0 & $\cdots$ & $x_{n} \alpha$ \\
\hline
\end{tabular}

TABLE 2. Fuzzy Context Table

$$
\tau=\left\{x_{0} \alpha, x_{1} \alpha, \ldots, x_{n} \alpha\right\} \subseteq \wp_{f}(Y) .
$$

In what follows a subset $\tau$ of $\wp(Y)$ will be called a fuzzy context on $Y$. Remark.

$A$ is a fuzzy relation into $Y$

$$
\begin{aligned}
& \leftrightarrow \quad A: I \rightarrow Y \text { is a fuzzy relation } \\
& \leftrightarrow A: I \times Y \rightarrow[0,1] \\
& \leftrightarrow A \in \wp_{f}(Y)
\end{aligned}
$$

\section{Armstrong's Inference Rules}

Armstrong's inference rules gives a basic framework of databases to treat the logical structure of dependencies on an attribute set. Let $A$ and $B$ be fuzzy relations into $Y$. A formal expression $A \triangleright B$, namely, an ordered pair of $A$ and $B$, is called a dependency on the attribute set $Y$.

Armstrong's Inference Rules
[A0] $\overline{A \triangleright A}$
[A1] $\frac{A \triangleright B}{A \sqcup C \triangleright B}$
[A2] $\frac{A \triangleright B \quad B \sqcup C \triangleright D}{A \sqcup C \triangleright D}$
$\left[\mathrm{A} 0{ }^{\prime}\right] \frac{A \sqsupseteq B}{A \triangleright B}$
$\left[\mathrm{A1}^{\prime}\right] \frac{A \triangleright B \quad C \sqsupseteq D}{A \sqcup C \triangleright B \sqcup D}$
$\left[\mathrm{A} 2^{\prime}\right] \frac{A \triangleright B \quad B \triangleright C}{A \triangleright C}$ 
Let $\mathcal{L}$ be a set of dependencies on $Y$. A derivation (or proof ) from $\mathcal{L}$ is a nonempty sequence

$$
\left\{A_{0} \triangleright B_{0}, A_{1} \triangleright B_{1}, \ldots, A_{m} \triangleright B_{m}\right\}
$$

of dependencies such that, for all $k=0,1, \ldots, m$, one of the following holds:

(1) $A_{k}=B_{k}([A 0])$ or $A_{k} \triangleright B_{k}$ is in $\mathcal{L}$,

(2) $\exists i<k$ such that:

(3) $\exists i, j<k$ such that:

$$
[A 1] \frac{A_{i} \triangleright B_{i}}{A_{k} \triangleright B_{k}}
$$

$$
[A 2] \frac{A_{i} \triangleright B_{i} A_{j} \triangleright B_{j}}{A_{k} \triangleright B_{k}}
$$

A dependency $A \triangleright B$ is provable from $\mathcal{L}$, written as $\mathcal{L} \vdash A \triangleright B$, if there is a derivation $\left\{A_{0} \triangleright B_{0}, A_{1} \triangleright B_{1}, \ldots, A_{m} \triangleright B_{m}\right\}$ from $\mathcal{L}$ such that $A=A_{m}$ and $B=B_{m}$. [A2]:

The system of inference rules [A0'], [A1'] and [A2'] is a mild variant of [A0], [A1] and

- $\left[A 0^{\prime}\right],\left[A 1^{\prime}\right],\left[A 2^{\prime}\right] \rightarrow[A 0],[A 1],[A 2]$

$\left[A 0^{\prime}\right] \frac{\overline{A \sqsupseteq A}}{A \triangleright A} \ldots[A 0]$

$$
\begin{aligned}
& {\left[A 1^{\prime}\right] \frac{A \triangleright B \overline{C \sqsupseteq 0_{I Y}}}{A \sqcup C \triangleright B} \ldots[A 1]} \\
& {\left[A 2^{\prime}\right] \frac{\left[A 1^{\prime}\right] \frac{A \triangleright B \overline{C \sqsupseteq C}}{A \sqsupseteq C \triangleright B \sqcup C} B \triangleright C \triangleright D}{A \sqcup C \triangleright D} \ldots[A 2] .}
\end{aligned}
$$

- $[A 0],[A 1],[A 2] \rightarrow\left[A 0^{\prime}\right],\left[A 1^{\prime}\right],\left[A 2^{\prime}\right]$

$$
\begin{aligned}
& \frac{[A 1] \frac{[A 0] \overline{B \triangleright B}}{B \sqcup A \triangleright B} \quad A \sqsupseteq B}{A \triangleright B} \ldots\left[A 0^{\prime}\right]
\end{aligned}
$$

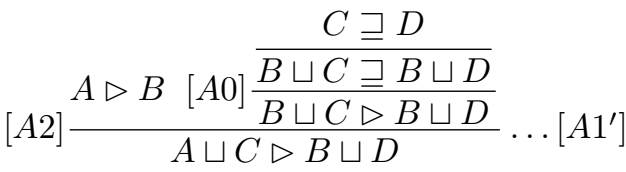

$$
\begin{aligned}
& \frac{[A 2] \frac{A \triangleright B[A 1] \frac{B \triangleright C}{B \sqcup A \triangleright C}}{A \sqcup A \triangleright C}}{A \triangleright C} \ldots\left[A 2^{\prime}\right]
\end{aligned}
$$

For example, the union rule

$$
[A 3] \frac{A \triangleright B A \triangleright C}{A \triangleright B \sqcup C}
$$

is proved from [A1'] and [A2'] as follows:

$$
\left[A 2^{\prime}\right] \frac{\left[A 1^{\prime}\right] \frac{A \triangleright B A \sqsupseteq A}{A \sqsupseteq A \sqcup B}\left[A 1^{\prime}\right] \frac{A \triangleright C B \sqsupseteq B}{A \sqsupseteq B \triangleright B \sqsupseteq C}}{A \triangleright B \sqsupseteq C}
$$

After all it makes no change to the provability for dependencies to add the rules [A0'], [A1'], [A2'] and [A3] into the formation rules of derivations.

Let $\mathcal{L}$ be a set of dependencies on $Y$ and $A$ a fuzzy relation into $Y$. Define a subset $\mathcal{L}_{A}$ of $\wp_{f}(Y)$ by

$$
\mathcal{L}_{A}=\{C: I \rightarrow Y \mid \mathcal{L} \vdash A \triangleright C\} .
$$

Lemma 4.1. Let $B$ be a finite fuzzy relation into $Y$. Then $\mathcal{L} \vdash A \triangleright B$ if and only if $B \sqsubseteq \sqcup \mathcal{L}_{A}$. 
Proof. $(\rightarrow)$ Assume $\mathcal{L} \vdash A \triangleright B$. Then $B \in \mathcal{L}_{A}$ by the definition of $\mathcal{L}_{A}$ and so $B \sqsubseteq \sqcup \mathcal{L}_{A}$.

$(\leftarrow)$ As $B$ is finite there is a finite subset $J \subseteq Y$ such that $B=\sqcup_{y \in J} B_{y}$. Assume $B \sqsubseteq \sqcup \mathcal{L}_{A}$ and let $y \in J$. Then we have $B_{y} \sqsubseteq \sqcup \mathcal{L}_{A}$ and

$$
\begin{aligned}
B_{y}(*, y) & =B(*, y) \\
& \leq \vee_{C \in \mathcal{L}_{A}} C(*, y) \\
& \doteq \max _{C \in \mathcal{L}_{A}} C(*, y)
\end{aligned}
$$

( $\doteq$ Because the set $[0,1]_{n}$ of fuzzy values is finite.) Hence

$$
\exists D \in \mathcal{L}_{A} \cdot B_{y}(*, y) \leq D(*, y),
$$

which implies $B_{y} \sqsubseteq D$. Hence we have $L \vdash A \triangleright B_{y}$, since

$$
\left\{A \triangleright D, D \triangleright B_{y}, A \triangleright B_{y}\right\}
$$

is a derivation from $\mathcal{L}$. Therefore $\mathcal{L} \vdash A \triangleright B$ holds by the union rule [A3], because $B=\sqcup_{y \in J} B_{y}$ is a finite union.

Remark. The above lemma always holds if the attribute set $Y$ is finite.

\section{Equivalence Relation}

In mathematical logic, equivalence relation is binary relation which is reflexive, symmetric, and transitive. In this definition, we use definition of indiscernibility relation that was introduced by Ganter and Wille[12]. We extend the definition of indiscernibility relation for fuzzy relation to define equivalence relation. We also show that the definition has the characteristics such as: reflexive, symmetric, and transitive. Definition of equivalence will be used to define fuzzy dependency in next section. The following definition is equivalence relation for general case(fuzzy and boolean case)

Definition 5.1. Let $A$ be a fuzzy relation into $Y$. Define an equivalence relation $\theta[A]$ on $\wp_{f}(Y)$ by

$$
(S, T) \in \theta[A] \leftrightarrow S \sqcap A=T \sqcap A .
$$

Note that $\theta[A]$ is crisp.

Proposition 5.2. Let $A$ and $B$ be fuzzy relation into $Y$. Then

(a) $\theta\left[\nabla_{I Y}\right]=i d_{\wp_{f}(Y)}$ and $\theta\left[0_{I Y}\right]=\nabla_{\wp_{f}(Y) \wp_{f}(Y)}$,

(b) $\theta[A \sqcup B]=\theta[A] \cap \theta[B]$,

(c) $\theta[A \sqcap B]=\theta[A] \theta[B]$.

Proof. (a-1)

$$
\begin{aligned}
(S, T) \in \theta\left[\nabla_{I Y}\right] & \leftrightarrow S \sqcap \nabla_{I Y}=T \sqcap \nabla_{I Y} \\
& \leftrightarrow S=T
\end{aligned}
$$

Since $S=T$ then we can conclude that $(S, S) \in \theta\left[\nabla_{I Y}\right]$ so we have $\theta\left[\nabla_{I Y}\right]=i d_{\wp_{f}(Y)}$ (a-2)

$$
\begin{aligned}
(S, T) \in \theta\left[\nabla_{\wp_{f}}(Y) \wp_{f}(Y)\right. & \rightarrow S \sqcap 0_{I Y}=T \sqcap 0_{I Y} \\
& \leftrightarrow(S, T) \in \theta\left[0_{I Y}\right]
\end{aligned}
$$

Then we can conclude that $\theta\left[0_{I Y}\right]=\nabla_{\wp_{f}(Y) \wp_{f}(Y)}$.

(b)

$$
\begin{aligned}
(S, T) \in \theta[A \sqcup B] & \leftrightarrow S \sqcap(A \sqcup B)=T \sqcap(A \sqcup B) \\
& \leftrightarrow\langle S \sqcap A=T \sqcap A\rangle \wedge\langle S \sqcap B=T \sqcap B\rangle \\
& \leftrightarrow\langle(S, T) \in \theta[A]\rangle \wedge\langle(S, T) \in \theta[B]\rangle \\
& \leftrightarrow(S, T) \in \theta[A] \cap \theta[B]
\end{aligned}
$$

Then we can conclude that $\theta[A \sqcup B]=\theta[A] \cap \theta[B]$. 
74 Akbar \& Mizoguchi, JMI Vol 15(2)Oct 2019, pp. 69-79 doi:10.24198/jmi.v15.n2.21693.69-79

(c)

$$
\begin{aligned}
(S, T) \in \theta[A \sqcap B] & \leftrightarrow S \sqcap(A \sqcap B)=T \sqcap(A \sqcap B) \\
& \stackrel{*}{\leftrightarrow} \exists U\langle S \sqcap A=U \sqcap A\rangle \wedge\langle U \sqcap B=T \sqcap B\rangle \\
& \leftrightarrow \exists U\langle(S, U) \in \theta[A]\rangle \wedge\langle(U, T) \in \theta[B]\rangle \\
& \leftrightarrow(S, T) \in \theta[A] . \theta[B]
\end{aligned}
$$

$\stackrel{*}{\leftarrow}$ :trivial.

$\stackrel{*}{\rightarrow}$ : Set $U=(S \sqcap A) \sqcup(T \sqcap B)$. Then we have

$$
\begin{aligned}
U \sqcap A & =((S \sqcap A) \sqcup(T \sqcap B)) \sqcap A \\
& =(S \sqcap A) \sqcup(T \sqcap B \sqcap A) \\
& =(S \sqcap A) \sqcup(S \sqcap B \sqcap A) \quad\{S \sqcap(A \sqcap B)=T \sqcap(A \sqcap B)\} \\
& =(S \sqcap A)
\end{aligned}
$$

\section{FUZZY IMPLICATION}

In formal concept analysis, implication is very important. Implication will construct concept latices of formal context. Using the definition logical implication in formal context, we will extend the definition in case of fuzzy formal context. We will define fuzzy implication then we will use it to show Armstrong's rule for fuzzy implication. We also show the soundness and completeness of fuzzy implication in the next section.

Definition 6.1. Let $\mathcal{T}$ be a fuzzy context on $Y, A \triangleright B$ a dependency on $Y$ and $\mathcal{L}$ a set of dependencies on $Y$, we define that fuzzy context table has fuzzy implication $\left(\mathcal{T} \models_{G}\right)$, with the definition:

(a) $\mathcal{T} \models_{G} A \triangleright B \leftrightarrow \forall T \in \mathcal{T} .\langle A \sqsubseteq T \rightarrow B \sqsubseteq T\rangle$,

(b) $\mathcal{T} \models_{G} \mathcal{L} \leftrightarrow \forall A \triangleright B \in \mathcal{L} . \mathcal{T} \models_{G} A \triangleright B$.

For a fuzzy context $\mathcal{T}$ on $Y$ we define another fuzzy context $\mathcal{T}^{*}$ on $Y$ by

$$
\mathcal{T}^{*}=\{\sqcap \mathcal{C} \mid \mathcal{C} \subseteq \mathcal{T}\} .
$$

Note. For example, $\{x \alpha \mid x \in X\}^{*}$ is the set of all formal concepts for a fuzzy relation $\alpha: X \rightarrow Y$.

Proposition 6.2. $\mathcal{T} \models_{G} A \triangleright B \leftrightarrow \mathcal{T}^{*} \models_{G} A \triangleright B$.

Proof. $(\leftarrow)$ It is trivial from $\mathcal{T} \subseteq \mathcal{T}^{*}$.

$(\rightarrow)$ Conversely assume $\mathcal{T} \models_{G} A \triangleright B$ and let $U=\sqcap \mathcal{C} \in \mathcal{T}^{*}$ for $\mathcal{C} \subseteq \mathcal{T}$. Then

$$
\begin{aligned}
A \sqsubseteq U & \leftrightarrow \forall C \in \mathcal{C} . A \sqsubseteq C & & \{U=\sqcap \mathcal{C}\} \\
& \leftrightarrow \forall C \in \mathcal{C} . A \sqsubseteq C & & \left\{C \in \mathcal{T}, \mathcal{T} \models_{G} A \triangleright B\right\} \\
& \leftrightarrow B \sqsubseteq U & & \{U=\sqcap \mathcal{T}\}
\end{aligned}
$$

which proves $\mathcal{T}^{*} \models_{G} A \triangleright B$.

Proof.

$$
\begin{aligned}
\mathcal{T} \models_{G} A \triangleright B & \leftrightarrow \forall \mathcal{T} .(A \sqsubseteq T) \rightarrow(B \sqsubseteq T) \\
& \stackrel{*}{\leftrightarrow} \forall \mathcal{C} \subseteq \mathcal{T} . \forall T \in \mathcal{C} .(A \sqsubseteq T) \rightarrow(B \sqsubseteq T) \\
& \leftrightarrow \forall \mathcal{C} \subseteq \mathcal{T} .(A \sqsubseteq \sqcap \mathcal{C}) \rightarrow(B \sqsubseteq \sqcap \mathcal{C}) \\
& \leftrightarrow \mathcal{T}^{*} \models_{G} A \triangleright B
\end{aligned}
$$


The implication dependencies also satisfy the Armstrong's inference rules. Next proposition, we would like to show the soundness of fuzzy implication on formal context.

Proposition 6.3. Let $\mathcal{T}$ be a fuzzy context on $Y$. Then
(a) $\mathcal{T} \models_{G} A \triangleright A$,
(b) If $\mathcal{T} \models_{G} A \triangleright B$, then $\mathcal{T} \models_{G} A \sqcup C \triangleright B$,
(c) If $\mathcal{T} \models_{G} A \triangleright B$ and $\mathcal{T} \models_{G} B \sqcup C \triangleright D$, then $\mathcal{T} \models_{G} A \sqcup C \triangleright D$.

Proof. (a) Trivial from definition.

(b) Assume $\mathcal{T} \models_{G} A \triangleright B$ and let $T \in \mathcal{T}$. Then

$$
\begin{aligned}
A \sqcup C \sqsubseteq T & \rightarrow A \sqsubseteq T \\
& \rightarrow B \sqsubseteq T .\left\{\mathcal{T} \models_{G} A \triangleright B\right\}
\end{aligned}
$$

Hence $\mathcal{T} \models_{G} A \sqcup C \triangleright B$.

(c) Assume $\mathcal{T} \models_{G} A \triangleright B$ and $\mathcal{T} \models_{G} B \sqcup C \triangleright D$. Let $T \in \mathcal{T}$. Then

$$
\begin{array}{rlrl}
A \sqcup C \sqsubseteq T & \leftrightarrow A \sqsubseteq T \wedge C \sqsubseteq T & \\
& \rightarrow B \sqsubseteq T \wedge C \sqsubseteq T & & \left\{\mathcal{T} \models_{G} A \triangleright B\right\} \\
& \leftrightarrow B \sqcup C \sqsubseteq T & & \\
& \rightarrow D \sqsubseteq T . & \left\{\mathcal{T} \models_{G} B \sqcup C \triangleright D\right\}
\end{array}
$$

Hence $\mathcal{T} \models_{G} A \sqcup C \triangleright D$.

Proposition 6.4. Let $\mathcal{T}_{0}=\{A\}$ be a particular fuzzy context on $Y$ such that $A \neq \nabla_{I} Y$. Then (a) $\mathcal{T}_{0} \models_{G} 0_{I Y} \triangleright C \leftrightarrow C \sqsubseteq A$,

(b) $\mathcal{T}_{0}=_{G} C \triangleright \nabla_{I Y} \leftrightarrow C \nsubseteq A$.

Proof. (a) $\mathcal{T}_{0} \models_{G} 0_{I Y} \triangleright C \leftrightarrow\left(0_{I Y} \sqsubseteq A \rightarrow C \sqsubseteq A\right) \leftrightarrow C \sqsubseteq A$.

(b) $\mathcal{T}_{0} \models_{G} C \triangleright \nabla_{I Y} \leftrightarrow\left(C \sqsubseteq A \rightarrow \nabla_{I Y} \sqsubseteq A\right) \leftrightarrow C \nsubseteq A$.

\section{Fuzzy Functional Dependency}

In relational database theory, a functional dependency is a constraint between two sets of attributes in a relation from a database. Functional dependency is one of important topic in database theory. Using functional dependency, we can conclude all "superkeys" in database systems. Baixeries et al has been introduced model of functional dependency on FCA[4]. We would like to extend his definition to fuzzy case.

Definition 7.1. Let $\mathcal{T}$ be a fuzzy context on $Y$ and $A \triangleright B$ a dependency on $Y$, we define that fuzzy context table has fuzzy implication $\left(\mathcal{T} \models_{F}\right)$, with the definition:

$$
\begin{aligned}
\mathcal{T} \models_{F} A \triangleright B & \leftrightarrow \forall S, T \in \mathrm{T} .\langle S \sqcap A=T \sqcap A \rightarrow S \sqcap B=T \sqcap B\rangle \\
& \leftrightarrow \forall S, T \in \mathrm{T} .\langle(S, T) \in \theta[A] \rightarrow(S, T) \in \theta[B]\rangle .
\end{aligned}
$$

Using Definition\%.1, we can investigate fuzzy functional dependency between attributes.

Lemma 7.2. Let $\mathcal{T}=\{S, T\}$ be fuzzy context on $Y$. then

$$
\mathcal{T} \models_{F} A \triangleright B \leftrightarrow\langle S \sqcap A=T \sqcap A \rightarrow S \sqcap B=T \sqcap B\rangle
$$

The functional dependencies satisfy the Armstrong's inference rules. Next proposition, we would like to show the soundness of fuzzy functional dependency.

Proposition 7.3. Let $\mathcal{T}$ be a fuzzy context on $Y$. Then

$(a) \mathcal{T} \models_{F} A \triangleright A$,

(b) If $\mathcal{T} \models_{F} A \triangleright B$, then $\mathcal{T} \models_{F} A \sqcup C \triangleright B$,

(c) If $\mathcal{T} \models_{F} A \triangleright B$, then $\mathcal{T} \models_{F} B \sqcup C \triangleright D$, and $\mathcal{T} \models_{F} A \sqcup C \triangleright D$, 
Proof. (a) It is trivial.

(b) Assume $\mathcal{T} \models_{F} A \triangleright B$ and $C \in \wp_{f}(Y)$.Then for all $S, T \in \mathcal{T}$ we have

$$
\begin{array}{rrr} 
& S \sqcap(A \sqcup C)=T \sqcap(A \sqcup C) & \\
\rightarrow & S \sqcap A=T \sqcap A & \{(A \sqcup C) \sqcap A=A\} \\
\rightarrow & S \sqcap B=T \sqcap B & \left\{\mathcal{T} \models_{F} A \triangleright B\right\}
\end{array}
$$

which proves $\mathcal{T} \models_{F} A \sqcup C \triangleright B$.

(c) Assume $\mathcal{T} \models_{F} A \triangleright B$ and $\mathcal{T} \models_{F} B \sqcup C \triangleright D$. Then for all $S, T \in \mathcal{T}$ it holds that

$$
\begin{aligned}
& S \sqcap(A \sqcup C)=T \sqcap(A \sqcup C) \\
& \leftrightarrow\langle S \sqcap A=T \sqcap A\rangle \wedge\langle S \sqcap C=T \sqcap C\rangle \\
& \rightarrow\langle S \sqcap B=T \sqcap B\rangle \wedge\langle S \sqcap C=T \sqcap C\rangle \quad\left\{T \models_{F} A \triangleright B\right\} \\
& \leftrightarrow S \sqcap(B \sqcup C)=T \sqcap(B \sqcup C) \\
& \rightarrow S \sqcap D=T \sqcap D, \\
& \left\{\mathcal{T} \models{ }_{F}(B \sqcup C) \triangleright D\right\}
\end{aligned}
$$

which proves $T \models_{F} A \sqcup C \triangleright D$.

After we showed the soundness of fuzzy functional dependency, we would like to define some condition of fuzzy functional dependency. The definition is important to show the completeness. We will show the completeness in the next section using the definition.

Definition 7.4. Consider a particular fuzzy context $\mathcal{T}_{0}=\left\{A, \nabla_{I Y}\right\}$ on $Y$ such that $A \neq \nabla_{I Y}$. Then for all fuzzy relations $C$ into $Y$ the following holds:

(a) $T_{0}={ }_{F} 0_{I Y} \triangleright C \leftrightarrow C \sqsubseteq A$,
(b) $T_{0} \models C \triangleright \nabla_{I Y} \leftrightarrow C \nsubseteq A$.

Proof. (a)

$$
\begin{aligned}
& \mathcal{T} \models{ }_{F} 0_{I Y} \triangleright C \\
& \leftrightarrow\left\langle\left(A, \nabla_{I Y}\right) \in \theta\left[0_{I Y}\right]\right\rangle \rightarrow\left\langle\left(A, \nabla_{I Y}\right) \in \theta[C]\right\rangle \\
& \leftrightarrow\left(A, \nabla_{I Y}\right) \in \theta[C] \\
& \leftrightarrow A \sqcap C=\theta_{I Y} \sqcap C=C \\
& \leftrightarrow C \sqsubseteq A \\
& \left\{\theta\left[0_{I Y}\right]=\nabla_{\wp_{f}(Y) \wp_{f}(Y)}\right\} \\
& \left\{C \sqsubseteq \nabla_{I Y}\right\}
\end{aligned}
$$

(b)

$$
\begin{aligned}
& \mathcal{T} \models_{F} \nabla_{I Y} \triangleright C \\
\leftrightarrow & \left\langle\left(A, \nabla_{I Y}\right) \in \theta[C]\right\rangle \rightarrow\left\langle\left(A, \nabla_{I Y}\right) \in \theta\left[0_{I Y}\right]\right\rangle \\
\leftrightarrow & \left\langle\left(A, \nabla_{I Y}\right) \notin \theta[C]\right\rangle \wedge\left\langle A=\nabla_{I Y}\right\rangle \\
\leftrightarrow & \left(\left(A, \nabla_{I Y}\right) \notin \theta[C]\right. \\
\leftrightarrow & C \nsubseteq A
\end{aligned}
$$

$$
\begin{array}{r}
\left\{\theta\left[\nabla_{I Y}\right]=\nabla_{\wp_{f}(Y)}\right\} \\
\left\{A \neq \nabla_{I Y}\right\} \\
\{\text { proof of }(\mathrm{a})\}
\end{array}
$$

\section{Completeness}

Now we will state the soundness and the completeness theorems of functional and implication dependencies for fuzzy contexts.

Theorem 8.1. Let $\mathcal{L}$ a set of dependencies and $A \triangleright B$ a dependency on a finite set $Y$. Then the following equivalence holds:

$$
\mathcal{L} \vdash A \triangleright B \leftrightarrow \mathcal{T} \subseteq \wp_{f}(Y) .\left(\mathcal{T}=_{\bullet} \mathcal{L} \rightarrow \mathcal{T} \models . A \triangleright B\right),
$$

where $\bullet=F$ or $G$. 
Proof. ( $\rightarrow$ ) (soundness) Assume $\mathcal{L} \vdash A \triangleright B$ and $\mathcal{T} \models$. $\mathcal{L}$. Then $\mathcal{T} \models \bullet A \triangleright B$ holds, because of the basic facts (A0), (A1) and (A2) in 5.5 and 6.3.

$(\leftarrow)$ (completeness) Assume $\forall \mathcal{T} .(\mathcal{T} \models . \mathcal{L} \rightarrow \mathcal{T} \models . A \triangleright B)$.

(I) In the case of $\sqcup \mathcal{L}_{A}=\nabla_{I Y}$.

$$
\begin{aligned}
\sqcup \mathcal{L}_{A}=\nabla_{I Y} & \rightarrow B \sqsubseteq \sqcup \mathcal{L}_{A} \\
& \leftrightarrow \mathcal{L} \vdash A \triangleright B
\end{aligned}
$$

(II) In the case of $\sqcup \mathcal{L}_{A} 6=\nabla_{I Y}$. By 5.6 and 6.4 we can choose a fuzzy context $\mathcal{T}_{0}$ satisfying the following conditions:

(a) $\mathcal{T}_{0}=.0_{I Y} \triangleright C \leftrightarrow C \sqsubseteq \sqcup \mathcal{L}_{A}$,

(b) $\mathcal{T}_{0}=. C \triangleright \nabla_{I Y} \leftrightarrow C \nsubseteq \sqcup \mathcal{L}_{A}$.

Then we will see $\mathcal{T}_{0}=$. $\mathcal{L}$, that is, $\mathcal{T}_{0} \models$. $C \triangleright D$ for all $C \triangleright D \in \mathcal{L}$.

(II-i) In the case of $C \sqsubseteq \sqcup \mathcal{L}_{A}$.

$$
\begin{aligned}
& C \sqsubseteq \sqcup \mathcal{L}_{A} \leftrightarrow \quad \mathcal{L} \vdash A \triangleright C \\
& \rightarrow \quad \mathcal{L} \vdash A \triangleright D \\
& \leftrightarrow \quad D \sqsubseteq \sqcup \mathcal{L}_{A} \\
& \leftrightarrow \quad \mathcal{T}_{0} \models \bullet 0_{I Y} \triangleright D \\
& \rightarrow \quad \mathcal{T}_{0} \models \text { • } C \triangleright D \\
& \left\{C \triangleright D \in \mathcal{L},\left[A 2^{\prime}\right]\right\} \\
& \left\{\left(A 0^{\prime}\right) \mathcal{T}_{0} \models \bullet C \triangleright 0_{I Y},\left(A 2^{\prime}\right)\right\}
\end{aligned}
$$

(II-ii) In the case of $C \nsubseteq \sqcup \mathcal{L}_{A}$.

\section{COMPARISON}

After we define fuzzy implication and fuzzy functional dependency. In this section, we would like to show the comparison between an fuzzy implication and a functional dependency. The following example will show the difference between fuzzy implication and functional dependency.

Example 9.1. Let $X=\left\{x_{1}, x_{2}, x_{3}, x_{4}, x_{5}\right\}$ be a domain of objects, $Y=\left\{y_{1}, y_{2}, y_{3}, y_{4}\right\}$ be a domain of attributes. Consider $\alpha: X \rightarrow Y$ be a fuzzy relation defined by Table 3. We assume $A=\left\{\left(y_{1}, 0.9\right)\right\}, B=\left\{\left(y_{3}, 0.5\right)\right\}, C=\left\{\left(y_{2}, 0.9\right)\right\}, D=\left\{\left(y_{4}, 0.4\right)\right\}$ be fuzzy relations into $Y$ (i.e. $A, B, C, D \in \wp_{f}(Y)$ )

\begin{tabular}{|c|c|c|c|c|}
\hline & $y_{1}$ & $y_{2}$ & $y_{3}$ & $y_{4}$ \\
\hline$x_{1}$ & 0.5 & 0.5 & 0.4 & 0.4 \\
$x_{2}$ & 0.6 & 0.6 & 1 & 1 \\
$x_{3}$ & 0.1 & 0.7 & 1 & 1 \\
$x_{4}$ & 0.9 & 0.9 & 0.4 & 0.4 \\
$x_{5}$ & 0.7 & 0.7 & 0.2 & 0.2 \\
\hline
\end{tabular}

TABLE 3. Fuzzy Relation $\alpha: X \rightarrow Y$

From example we got:

(1) $\mathcal{T} \models_{F} A \triangleright B$ and $\mathcal{T} \nvdash_{G} A \triangleright B$

If we compare tuple in " $x 4 ", T_{4}$ then we get $A \sqsubseteq T_{4}$ but $B \nsubseteq T_{4}$. It means $\mathcal{T} \nvdash_{G} A \triangleright B$. But, all condition tuple will fulfill the condition of fuzzy functional dependency.

(2) $\mathcal{T} \models_{G} C \triangleright D$ and $\mathcal{T} \not{ }_{F} C \triangleright D$

In this condition, we can compare tuple in " $x 4$ " with tuple in " $x 2$ " or " $x 3$ ", then we have $C \sqcap T_{2} \neq C \sqcap T_{4}$ but $D \sqcap T_{2}=D \sqcap T_{4}$. Also, tuple in $x_{3}$ then $C \sqcap T_{3} \neq C \sqcap T_{4}$ but $D \sqcap T_{3}=D \sqcap T_{4}$. But for all condition will fulfill the condition of fuzzy implication.

Then we can conclude that: $\mathcal{T} \models_{F} A \triangleright B$ is not equivalent with $\mathcal{T} \models_{I} A \triangleright B$. Also, $\mathcal{T} \models_{I} C \triangleright D$ is not equivalent with $\mathcal{T} \models_{F} C \triangleright D$ 
Next, we would like to observe the equivalent condition of fuzzy functional and implication dependency. We review and extend the equivalent condition in the case of boolean[13] to the general or fuzzy relation case. Let $\mathcal{T}$ be a fuzzy context on $Y$. Define another fuzzy context $\overline{\mathcal{T}}$ on $Y$ by $\overline{\mathcal{T}}=\{(S \Rightarrow T) \sqcap(T \Rightarrow S) \mid S, T \in \mathcal{T}\}$.

Theorem 9.2. $\mathcal{T} \models_{F} A \triangleright B$ if and only if $\overline{\mathcal{T}} \models_{G} A \triangleright B$.

Proof. First we note that $S \sqcap A=T \sqcap A$ is equivalent with $(S \sqcap A \sqsubseteq T)$ and $(T \sqcap A \sqsubseteq S)$. By Lemma 2.1(b), we get $S \sqcap A=T \sqcap A$ is equivalent to $A \sqsubseteq(S \Rightarrow T) \sqcap(T \Rightarrow S)$.

For all $V \in \overline{\mathcal{T}}$ there exists a pair of fuzzy relations $S, T \in \mathcal{T}$ such that $V=(S \Rightarrow T) \sqcap(T \Rightarrow S)$. So we have $A \sqsubseteq V$ is equivalent with $S \sqcap A=T \sqcap A$.

$(\rightarrow)$ Since $\mathcal{T} \models_{F} A \triangleright B$, if $S \sqcap A=T \sqcap A$ then $S \sqcap B=T \sqcap B$. Then if $A \sqsubseteq V$ then $B \sqsubseteq V$ which means $\overline{\mathcal{T}} \models_{G} A \triangleright B$.

$(\leftarrow)$ Since $\overline{\mathcal{T}} \models_{G} A \triangleright B$, we get if $A \sqsubseteq V$ then $B \sqsubseteq V$. Then if $S \sqcap A=T \sqcap A$ then $S \sqcap B=T \sqcap B$ which means $\mathcal{T} \models_{F} A \triangleright B$.

Theorem 9.3. (a) If $\nabla_{I Y} \in \mathcal{T}$, then $\mathcal{T} \models_{F} A \triangleright B$ implies $\mathcal{T} \models_{G} A \triangleright B$.

(b) If $\mathcal{T} \sqsubseteq \overline{\mathcal{T}} \sqsubseteq \mathcal{T}^{*}$, then $\mathcal{T} \models_{G} A \triangleright B$ if and only if $\mathcal{T} \models_{F} A \triangleright B$.

Proof. $\quad$ (a) Assume $\nabla_{I Y} \in \mathcal{T}$, then $\mathcal{T} \sqsubseteq \overline{\mathcal{T}}$, because $\left(S \Rightarrow \nabla_{I Y}\right) \sqcap\left(\nabla_{I Y} \Rightarrow S\right)=S$ for $S \in \mathcal{T}$. Since $\mathcal{T} \models_{F} A \triangleright B$, we have $\overline{\mathcal{T}} \models_{G} A \triangleright B$ by Theorem 9.2.

(b) $(\leftarrow)$ Since $\mathcal{T} \sqsubseteq \overline{\mathcal{T}}$ then we proves if $\overline{\mathcal{T}} \models_{G} A \triangleright B$ then $\mathcal{T} \models_{G} A \triangleright B$.

$(\rightarrow)$ From Proposition 6.2 we have $\mathcal{T}^{*} \models_{G} A \triangleright B$. Since $\overline{\mathcal{T}} \sqsubseteq \mathcal{T}^{*}$ we have $\overline{\mathcal{T}} \models_{G} A \triangleright B$. So we proved that $\overline{\mathcal{T}} \models_{G} A \triangleright B$. By Proposition 9.2 we know that $\overline{\mathcal{T}} \models_{G} A \triangleright B$ is equivalent to $\mathcal{T} \models_{F} A \triangleright B$. Then we proved $\mathcal{T} \models_{G} A \triangleright B$ if and only if $\mathcal{T} \models_{F} A \triangleright B$.

Using theorem 9.2 and 9.3 can be used to find dependency using the properties then we can reduced the dataset using the theorems.

\section{Conclusion}

In this paper, we extend the idea that Armstrong inference rules are soundness and completeness for fuzzy functional and implication dependencies. We proved some properties about a dependency and an implication. After that, we give an example to explain comparison between a fuzzy implication and a fuzzy functional dependency. In the common condition, we can not make fuzzy rules(implication) using attribute dependency, and also vice versa. But, we also gave a condition that a fuzzy implication and a functional dependency are equivalent and showed that a functional dependency can be reduced to an implication when the condition of table(data) is fulfill in theorem 9.2 and 9.3 .

Future works include to construct a theory of fuzzy relational database theory with computer verified formal proofs using relational calculus.

\section{REFERENCES}

[1] Mohammad Deni Akbar and Yoshihiro Mizoguchi. A formalization of a fuzzy relational database model using relational calculus. Bulletin of Informatics and Cybernetics, 48(6):83-104, 2016.

[2] Mohammad Deni Akbar and Yoshihiro Mizoguchi. Formal equivalence classes model of fuzzy relational databases using relational calculus. 2017 International Conference on Applied Computer and Communication Technologies (ComCom), pages 1-6, 2017.

[3] Mohammad Deni Akbar, Yoshihiro Mizoguchi, and Adiwijaya. Class dependency of fuzzy relational database using relational calculus and conditional probability. Journal of Physics: Conference Series, 971(1):012001, 2018

[4] Jaume Baixeries, Mehdi Kaytoue, and Amedeo Napoli. Characterizing functional dependencies in formal concept analysis with pattern structures. Annals of Mathematics and Artificial Intelligence, 72(1):129-149, Oct 2014 
[5] Catriel Beeri, Ronald Fagin, and John H. Howard. A complete axiomatization for functional and multivalued dependencies in database relations. In Proceedings of the 1977 ACM SIGMOD International Conference on Management of Data, SIGMOD '77, pages 47-61, New York, NY, USA, 1977. ACM.

[6] Radim Belohlavek, Pablo Cordero, Manuel Enciso, Ángel Mora, and Vilem Vychodil. Automated prover for attribute dependencies in data with grades. Int. J. Approx. Reasoning, 70(C):51-67, March 2016.

[7] Radim Belohlávek and Vilém Vychodil. Semantic entailment of attribute-dependency formulas and their non-redundant bases. In JCIS, 2006.

[8] Radim Belohlavek and Vilem Vychodil. Codd's relational model from the point of view of fuzzy logic. Journal of Logic and Computation, 21(5):851, 2011.

[9] B. Bhuniya and P. Niyogi. Lossless join property in fuzzy relational databases. Data \& Knowledge Engineering, 11(2):109 - 124, 1993

[10] E. F. Codd. A relational model of data for large shared data banks. Commun. ACM, 13(6):377-387, jun 1970.

[11] Ivo Duntsch and Gunther Gediga. Uncertainty measures of rough set prediction. Artificial Intelligence, 106(1):109 - 137, 1998.

[12] Bernhard Ganter and Rudolf Wille. Formal Concept Analysis: Mathematical Foundations. Springer-Verlag New York, Inc., Secaucus, NJ, USA, 1st edition, 1997.

[13] Toshikazu Ishida, Kazumasa Honda, and Yasuo Kawahara. Implication and functional dependency in intensional contexts. Bulletin of informatics and cybernetics, 40:101-111, dec 2008.

[14] Michinori Nakata. Formulation of Division Operators in Fuzzy Relational Databases, pages 144-156. Physica-Verlag HD, Heidelberg, 2000.

[15] H. Okuma and Y. Kawahara. Relational aspects of relational database dependencies. Bulletin of Informatics and Cybernetics, 16:91-104, 2000.

[16] K. V. S. V. N. Raju and Arun K. Majumdar. Fuzzy functional dependencies and lossless join decomposition of fuzzy relational database systems. ACM Trans. Database Syst., 13(2):129-166, June 1988.

[17] Motohide Umano and Satoru Fukami. Fuzzy relational algebra for possibility-distribution-fuzzy-relational model of fuzzy data. J. Intell. Inf. Syst., 3(1):7-27, February 1994.

[18] Rudolf Wille. Restructuring lattice theory: an approach based on hierarchies of concepts. In Ivan Rival, editor, Ordered Sets, pages 445-470. Reidel, 1982.

[19] Lofti A. Zadeh. Fuzzy sets. Information and Control, 8:338-353, 1965. 
